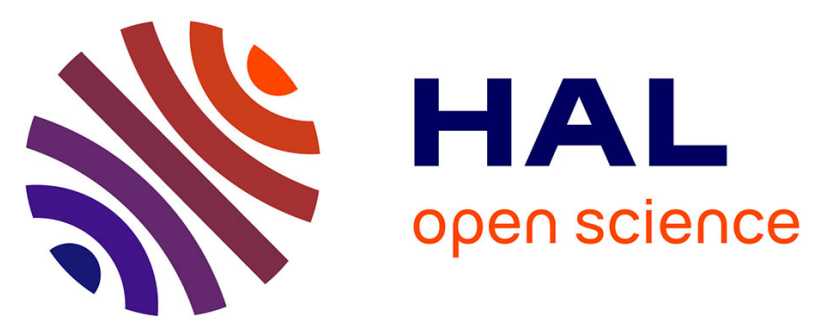

\title{
Determination of the efficiency of high purity germanium and silicon diode detectors for improved assessment of emission spectra delivered by medical X-ray tubes
}

Sybelle Deloule, Johann Plagnard, Marc Denozière, Isabelle Aubineau-Lanièce

\section{To cite this version:}

Sybelle Deloule, Johann Plagnard, Marc Denozière, Isabelle Aubineau-Lanièce. Determination of the efficiency of high purity germanium and silicon diode detectors for improved assessment of emission spectra delivered by medical X-ray tubes. X-Ray Spectrometry, 2013, 42 (4), pp.201 - 206. $10.1002 / x r s .2471$. cea-01791938

\section{HAL Id: cea-01791938 https://hal-cea.archives-ouvertes.fr/cea-01791938}

Submitted on 12 Feb 2019

HAL is a multi-disciplinary open access archive for the deposit and dissemination of scientific research documents, whether they are published or not. The documents may come from teaching and research institutions in France or abroad, or from public or private research centers.
L'archive ouverte pluridisciplinaire HAL, est destinée au dépôt et à la diffusion de documents scientifiques de niveau recherche, publiés ou non, émanant des établissements d'enseignement et de recherche français ou étrangers, des laboratoires publics ou privés. 


\title{
Determination of the efficiency of high purity germanium and silicon diode detectors for improved assessment of emission spectra delivered by medical X-ray tubes
}

\author{
Sybelle Deloule,* Johann Plagnard, Marc Denoziere and \\ Isabelle Aubineau-Laniece
}

\begin{abstract}
X-ray sources are widely used in medicine: brachytherapy, radiodiagnosis, mammography and contact radiotherapy. Kerma in air is the primary quantity measured to determine the dose to the patient. Accurate air kerma assessment is obtained using correction factors calculated using the emission spectrum. The Laboratoire National Henri Becquerel launched an in-depth study of the spectral emission of its reference X-ray beams used in dosimetry. Two semiconductor detectors are discussed here: a High-Purity germanium and a silicon PiN, both cooled and operated with dedicated electronics and software. In the low energy range ( $E \mathbf{5 0} \mathrm{keV}$ ), those spectrometers are complementary but require a careful calibration to deduce the emitted spectrum from the detected one. Indeed, both detectors were characterized in terms of spectral response and intrinsic efficiency using a tuneable monochromatic X-ray source (SOLEX at CEA Saclay) in the 5- to 20-keV energy range and various radionuclides. The characterization methods and results, including the first measured spectra of medical X-ray tubes (high voltage $<50 \mathbf{~ k V}$ ), are presented in this work. This paper presents the first step of a broader project, aiming at assessing the emission spectrum independently of the detector choice. Copyright $\odot 2013$ John Wiley \& Sons, Ltd.
\end{abstract}

\section{Introduction}

Medical physicists assess the quality of X-ray tubes by measuring the half value layer, which is the thickness of a given material (aluminum or copper) needed to divide by two the air kerma at a given distance. However, this criterion is not accurate enough, and the French metrology laboratory aims at a better knowledge of the quality of its reference beams by spectrometric measurements. The high emission fluence rate of some medical devices as mammographs (up to $10^{8}$ photons $\mathrm{s}^{-1} \mathrm{~mm}^{-2}$ ) is one of the main limits to the use of semiconductors, as the electronics generate pile up. Reducing the solid angle by collimation is necessary; nevertheless, correct alignment with the beam becomes vital to avoid measuring a scattered spectrum. In the low energy range, silicon and germanium offer a good energy resolution, but silicon efficiency drops after $10 \mathrm{keV}$ for a $500 \mu \mathrm{m}$ thick crystal, and germanium $\mathrm{K}$ absorption edge induces peak shape change above $11 \mathrm{keV}$ because of the cross-section edge. The method proposed here takes advantage of both detectors, by studying their response function to correct measured spectra for the detector's response. This paper presents the characterization of the selected semiconductors (a high purity germanium and a silicon diode), in terms of shape and efficiency. Then a first example of spectrum correction is detailed and perspectives are discussed.

\section{Material and methods}

Two semiconductor detectors were characterized in terms of peak shape and detection efficiency in the 5- to 60-keV energy range at the Laboratoire National Henri Becquerel (LNHB).

\section{Detectors}

The first one is a silicon PiN crystal of small volume $(3.6 \times 3.6$ $0.5 \mathrm{~mm}^{3}$ ), cooled by two Peltier stages. As its detection efficiency drops above $10 \mathrm{keV}$, it is dedicated to spectral measurement below $30 \mathrm{keV}$. The second one, a high purity germanium (Ge) crystal, is bulkier, $\left(100 \mathrm{~mm}^{2} \times 10 \mathrm{~mm}\right)$, resulting in a detection efficiency above $10 \%$ in the whole range $[5-300 \mathrm{keV}]$. It is electrically cooled down to $87 \mathrm{~K}$ by a cryo-generator.

Both are set up with dedicated digital signal processor, electronics and software. The main characteristics of the two systems are given in Table 1.

\section{Calibration sources}

For energies above $10 \mathrm{keV}$, four sealed sources $\left({ }^{241} \mathrm{Am},{ }^{133} \mathrm{Ba}\right.$, ${ }^{152} \mathrm{Eu}$ and $\left.{ }^{125} \mathrm{I}\right)$, of about $1 \mathrm{MBq}$ each, were used. Those sources were made and calibrated at LNHB-LMA, the French metrological reference laboratory for activity. A dedicated bench was set up, to guaranty the source-detector alignment and set precisely the solid angle needed for intrinsic efficiency assessment.

A monochromatic X-ray source, the SOLEX facility at LNHB$\mathrm{LMA}^{[1,2]}$ was used to study both detectors response at energies below $17 \mathrm{keV}$ and, in particular, the germanium $\mathrm{K}$ edge $(11.1 \mathrm{keV})$. SOLEX operates in a vacuum assembly as follows: $\mathrm{A}$

* Correspondence to: Sybelle Deloule, CEA, LIST, Laboratoire National Henri Becquerel, 91191 Gif sur Yvette, France.E-mail: Sybelle.DELOULE@cea.fr

CEA, LIST, Laboratoire National Henri Becquerel, 91191 Gif sur Yvette, France 
Table 1. Nominal characteristics of the detectors

\begin{tabular}{|lccccccc|} 
& $Z$ & $\begin{array}{c}\text { Surface } \\
\text { area }\left(\mathrm{mm}^{2}\right)\end{array}$ & $\begin{array}{c}\text { thickness } \\
(\mathrm{mm})\end{array}$ & $\begin{array}{c}90 \%<\text { efficiency } \\
<100 \%(\mathrm{keV})\end{array}$ & $\begin{array}{c}\text { FWHM at } \\
5.9 \mathrm{keV}\end{array}$ & $\begin{array}{c}\text { Be window } \\
(\mu \mathrm{m})\end{array}$ & $\begin{array}{c}\text { Operating } \\
\text { temperature }(\mathrm{K})\end{array}$ \\
\hline Silicon & 14 & 13 & 0.50 & {$[3-10]$} & 200 & 25 & 254 \\
Germanium & 32 & 100 & 10 & {$[3-100]$} & 145 & 87 \\
\hline FWHM, full width at half maximum.
\end{tabular}

windowless X-ray tube produces a polychromatic spectrum. Then, a monochromator crystal, working in the reflexion or transmission modes (Bragg's law), selects the energy ( $\triangle \mathrm{E}-50 \mathrm{eV})$. Two crystals were needed to cover the range of interest: LiF (200) [3.6-12.5 keV] and quartz (1340) [6.2-20.8 keV]. The tube operates up to $20 \mathrm{kV}$ and $100 \mathrm{~mA}$ and has a copper anode. The flux and the size of the output beam can be adjusted with $X-Z$ motorized slits. The flux is monitored with a calibrated reference proportional counter ( $\mathrm{Ar} 90 \%-\mathrm{CH}_{4}$ 10\%; $500 \mathrm{hPa}$ ) whose absolute efficiency $\left(\right.$ Eff $\left._{\text {ref }}\right)$ had been previously determined. ${ }^{[3]}$ Shape response and detection efficiency drop were studied using an X-ray beam perpendicular to the detector surface. Unlike the smaller silicon detector, the germanium detector was not directly connected to the SOLEX assembly, operating under vacuum, but behind a beryllium window in air. This set-up difference induced corrections for beam transmission in air $(7.0 \mathrm{~cm})$ and beryllium $(100 \mu \mathrm{m})$.

\section{Experimental protocol and data analysis}

Because the study was not only focussed on the shape of the spectrum but also on its area, determination of the absolute detection efficiency of both systems was a mandatory step of the project. Both detectors efficiency $\left(E f_{\text {det }}\right)$ were measured at SOLEX, using its patented reference proportional counter (noted ref) according to the formula:

$$
E f f_{\mathrm{det}}=\frac{N_{\mathrm{det}} * t_{\mathrm{ref}} * E f f_{\mathrm{ref}}}{N_{\mathrm{ref}} * t_{\mathrm{det}} * T}
$$

where $N$ is the number of counts in the region of interest, $t$ is the acquisition time and $T$ is the transmission factor within air and beryllium ( $T=1$ for the silicon detector).

Total absorption and escape peak shapes were determined using the convolution software COLEGRAM to fit Gaussian, tailed Gaussian and Hypermet functions to the peaks. ${ }^{[4,5]}$ To ease the fitting process, the software allows us to set parameters like full width at half maximum. As an example, Fig. 1 displays the fit of a Hypermet function to measurement points in the total absorption peak range. Please note that in all figures, the total absorption peak is denoted as PE. By fitting those functions to the data, tail area and step area could be worked out using formula (2) and (3):

$$
\begin{gathered}
\text { Tail area }=\text { TailedGaussian area }- \text { Gaussian area } \\
\text { Step area }=\text { Hypermet area }- \text { TailedGaussian area }
\end{gathered}
$$

Spectra are measured at a $40-\mathrm{cm}$ distance in air, at which $\mathrm{X}$-rays below $5 \mathrm{keV}$ are barely transmitted. Above $17 \mathrm{keV}$, the reference counter detection efficiency drops. Therefore, the study using SOLEX was limited to the 5- to 17-keV energy range.

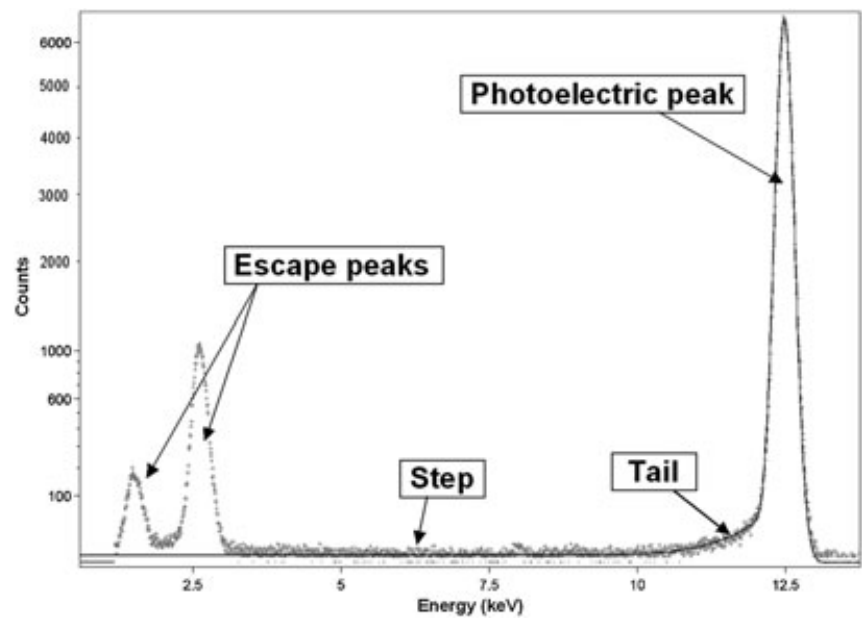

Figure 1. Example of spectrum analysis using the Hypermet function in COLEGRAM (germanium detector, SOLEX beam at $12.500 \mathrm{keV}, Y$-axis: square root scale).

By using radionuclides $\left({ }^{241} \mathrm{Am},{ }^{133} \mathrm{Ba},{ }^{152} \mathrm{Eu}\right.$ and $\left.{ }^{125} \mathrm{I}\right)$, both detectors were calibrated for more than 20 additional energies between 13 and $300 \mathrm{keV}$.

A precise calibration is then possible, and the response function of the detector can be modeled.

\section{Results}

\section{Resolution}

The final project aims at deducing spectra really emitted from measured spectra. Therefore, energy resolution widening induced by the electronics has to be precisely known as a component of the response function.

The Gaussian-fitted total absorption peak is defined by its energy $E_{0}$, its amplitude $A$ (proportional to its surface area) and its standard width $\sigma$, expressed as

$$
\sigma^{2}=K+F * \omega * E=(\mathrm{FWHM} / 2.355)^{2}
$$

where $\sqrt{K}$ is a constant related to the electrical noise and charge collection problems; $\omega$ is the pair creation energy; $F$ is the material-dependent Fano factor and $E$ is the energy of the vincident photon.

Given $\omega_{\mathrm{Si}}=3.62 \mathrm{eV}$ and $\omega_{\mathrm{Ge}}=2.96 \mathrm{eV}$, the $F$ and $\sqrt{K}$ values were experimentally assessed from the slope and the ordinate at origin as drawn in Fig. 2. For germanium, it was found that $F=0.110$ and $\sqrt{K}=67 \mathrm{eV}$, whereas for silicon, $F=0.141$ and $\sqrt{K}=136 \mathrm{eV}$. Those results show clearly that the germanium presents a better energy resolution than the silicon, which is coherent with previous measurements on similar detectors. 


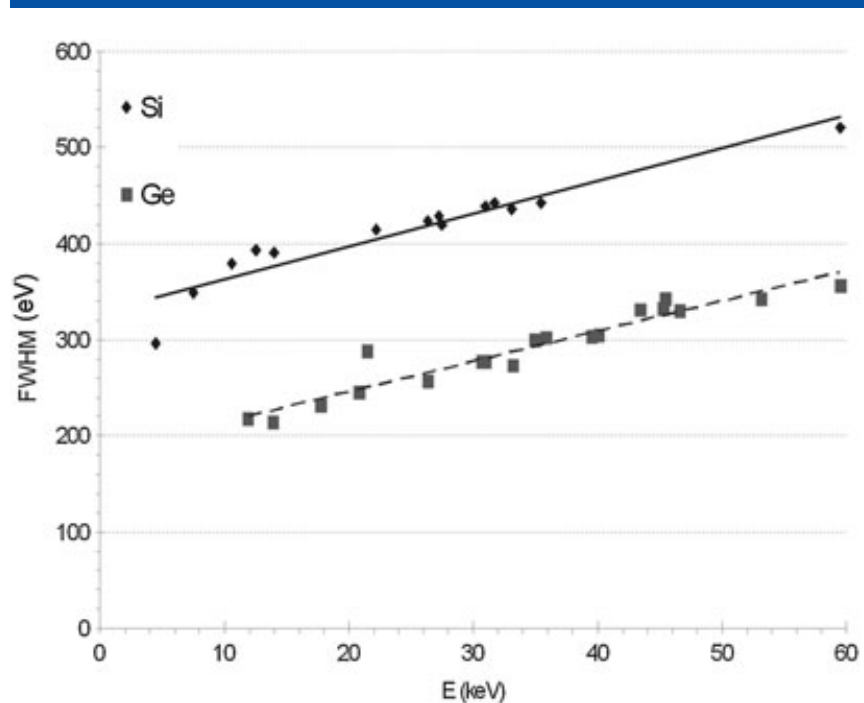

Figure 2. Full width at half maximum (FWHM) of silicon and germanium detectors with sealed sources (Am, Ba, Eu and I).

\section{Peak shape}

Usually, the total absorption peak (PE) is fitted by a Gaussian, as it is often the only available option in commercial software. Nevertheless, charge collection defaults escape in and out the detector, and dead layer's absorption can distort the Gaussian peak shape. This is especially true when the interaction depth is small, that is, at low energy or nearby absorption edges. The region of interest being above $5 \mathrm{keV}$, only the germanium $K$ edge (at $11.103 \mathrm{eV}$ ) was of particular interest. After the germanium $K$ edge, mass attenuation coefficient increases dramatically, and the depth of interaction decreases so that 150 and $22 \mu \mathrm{m}$ of Germanium are, respectively, needed to absorb $90 \%$ of photons of 11.100 and of $11.125 \mathrm{keV}$. Therefore, more edge effects and charge collection problems are observed in this energy range. Earlier studies led to define this shape as a tailed Gaussian and sometimes to add a constant step on the left of the tailed Gaussian (Hypermet function). COLEGRAM software allows calculation of the tail and the step area of germanium near the $\mathrm{K}$ edge, to determine whether they are negligible or not.

Figure 3 plots, as a function of the incident photon energy, the tail area and the step area, respectively, normalized to the sum of the PE peak and its related escape peaks. Both effects being lower than $2 \%$, they were not considered in this first correction process

The thickness of the partial active layer (PAL) can be derived from the tail to PE peak area ratio, where charge collection is not complete because of electric field heterogeneity. The probability to create counts in the tail can be inferred from the area of the tail, the PE peak and escape peaks. Then the PAL's thickness was calculated from the Beer-Lambert law. ${ }^{[2,6]}$ The germanium measured has a thin PAL: $Z_{\mathrm{PAL}}=(0.137 \pm 0.030) \mu \mathrm{m}$, which is coherent with other publications.

\section{Escape peaks}

In Fig. 3, it is worth noticing the discontinuity at $11.1 \mathrm{keV}$, because of the germanium $\mathrm{K}$ edge. When the energy of the incident beam is above the binding energy of the $\mathrm{K}$ shell, electron ejection causes either X-ray fluorescence or Auger electron emissions during electronic rearrangement. The ratio of fluorescence on $K$ shell
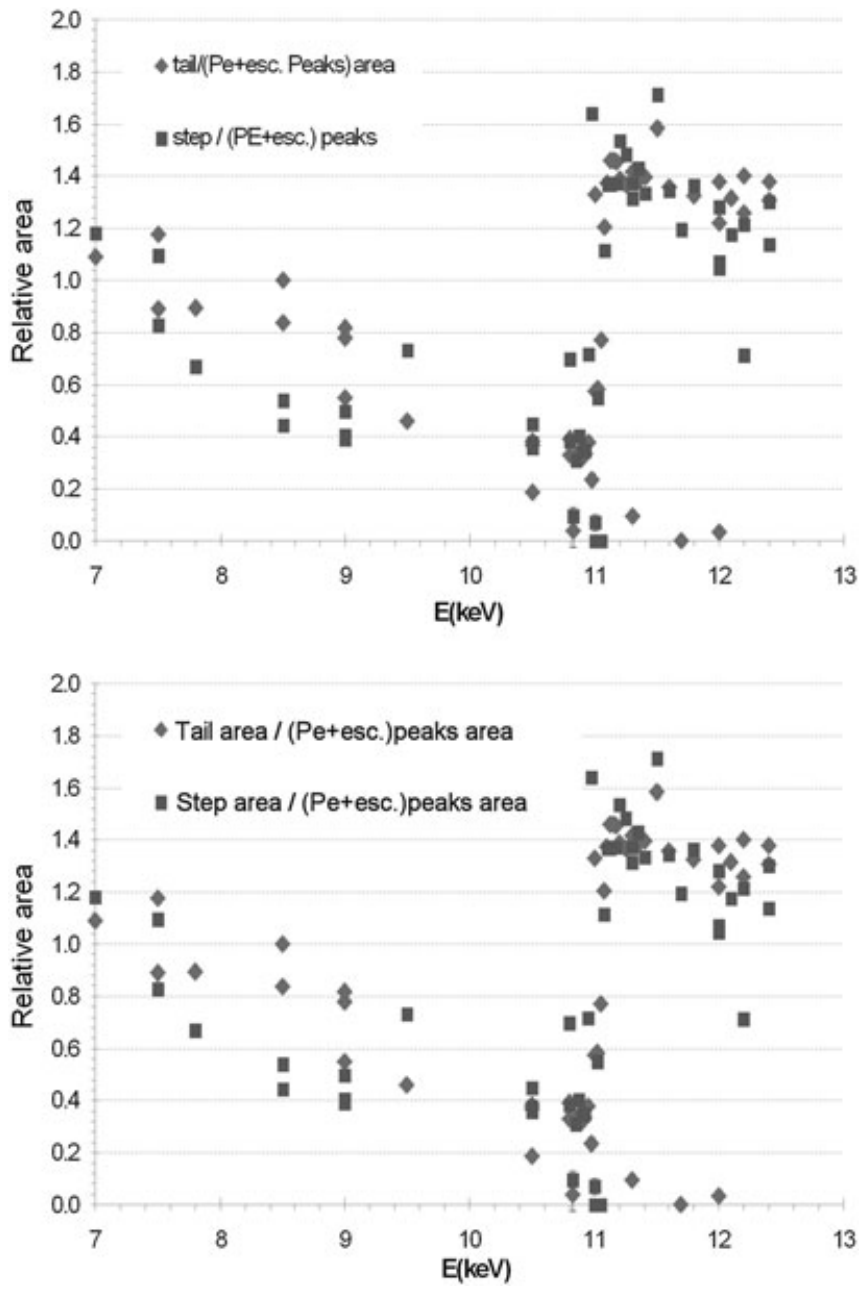

Figure 3. Step and tail to PE peak area ratios as functions of energy.

ionizations is $\omega_{\mathrm{K}}=0.535$. According to the energy diagram, we can have either $K_{\alpha}=9.875 \mathrm{keV}$ or $K_{\beta}=10.982 \mathrm{keV}$, and $K_{\alpha}$ is about 15 times more intense than $K_{\beta}$. When the depth of interaction is small, or close to the thickness of the detector, the fluorescence $\mathrm{X}$-rays may escape from the detector active volume, resulting in peaks at $E_{\mathrm{PE}}-K_{\alpha}$ and $E_{\mathrm{PE}}-K_{\beta}$. The probability of those escape peaks drops when incident energy rises as the interaction depth increases. This phenomenon was first studied by S. J.B. Reed and N. G. Ware for silicon diodes and S. R. Piermattei and M. P. Fioratti for germanium detectors. ${ }^{[7,8]}$ Assuming that the beam is perpendicular to the crystal surface, the following formulas express the ratio of escaped $K$ fluorescence photons $(\mathrm{Ne})$ to incident photons of energy $E$ that interacted by photoelectric effect $(N)$ was demonstrated:

$$
\frac{\mathrm{Ne}}{N}=0.5 \omega_{\mathrm{K}}\left(1-\frac{1}{\mathrm{r}}\right)\left[1-\frac{\mu\left(E_{\mathrm{K}}\right)}{\mu(E)} * \ln \left(1+\frac{\mu(E)}{\mu\left(E_{\mathrm{K}}\right)}\right)\right]
$$

For germanium, $E_{\mathrm{K}}$ is $K_{\alpha}$ energy and $r$ is the ratio of mass attenuation coefficient on both sides of the $K$ edge:

$$
r=\frac{\mu(11125)}{\mu(11100)}=\frac{197}{28.1}=7.011
$$


Although $N$ is not always known, the number of photons in the total absorption peak ( $\left.N_{\mathrm{PE}}\right)$ can be measured. Considering that the detector is thick enough and that Compton scattering is negligible, $N$ can be replaced in (5) by

$$
N=N_{\mathrm{PE}}+\mathrm{Ne}
$$

Figure 4 shows that there is a good agreement (within 10\%) between theoretical formula and measurement of the escape peaks sum to PE peak area ratio. Our results are also consistent with Christensen's. ${ }^{[9]}$ In addition, the measured ratio of $K_{\beta}$ to $K_{\alpha \iota}$ is close to 0.148 as given by Christensen ${ }^{[9]}$ :

$$
\frac{K_{\beta}}{K_{\alpha}}=0.147
$$

Moreover, as the expression (5) is more complex if the incident angle differs from $90^{\circ}$, measurement conditions have to be carefully chosen. Escape peaks cannot be neglected in the range of study. Because those peaks contribute to up to $18 \%$ of the PE peak area and are energy-dependent, escape phenomenon has to be corrected.

We fitted an exponential decay to the measured escape to PE peak area ratio in Fig. 4. Therefore, we could calculate for every energy bin the resulting escape peaks energy bins and relative intensities. We started from the highest channel, and calculated the two escape peak channels and their relative intensities, to add those counts to the total absorption peak, and subtracted them from lower energy bins. Then we reiterated this calculation channel by channel until we reached the $K$ edge energy, $E_{K}=11.103 \mathrm{keV}$, and then corrected all bins for efficiency.

This phenomenon occurs in silicon too, but as $E_{\mathrm{K}, \mathrm{Si}}=1.839 \mathrm{keV}$ and $\omega_{\mathrm{K}, \mathrm{Si}}=0.038$, the fluorescence $\mathrm{X}$-ray is less likely to escape. Indeed, escape peaks are less than $1 \%$ of the PE peak area, so no escape correction will be carried out for the silicon detector's spectra.

\section{Efficiency calibration}

Radionuclides and SOLEX are reference sources, allowing us to measure the intrinsic efficiency of both detectors. Figure 5(a)

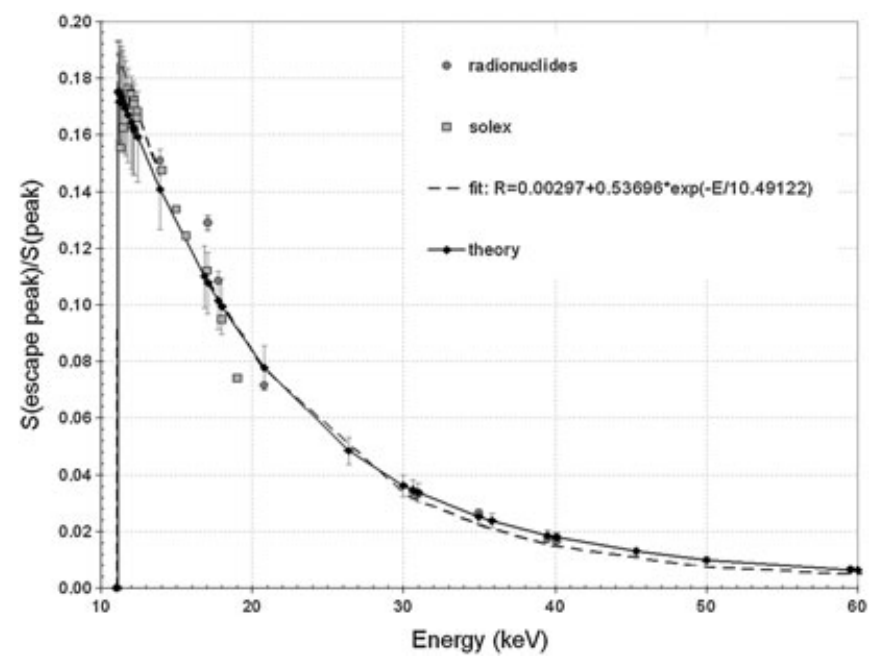

Figure 4. Escape to PE peak area ratio of the germanium detector (SOLEX and radionuclides' data).

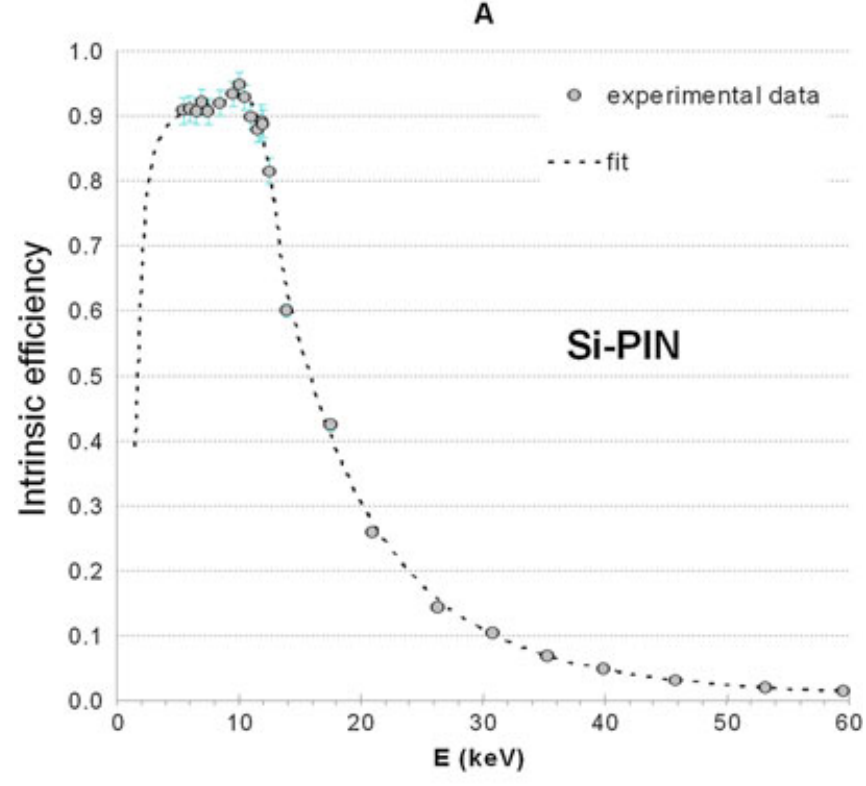

B

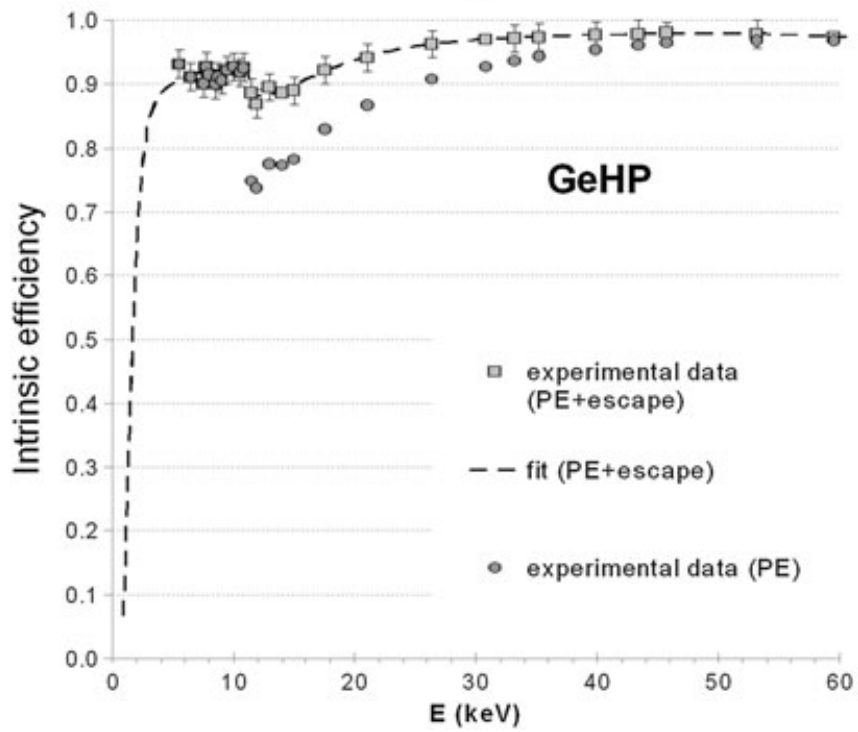

Figure 5. (a) and (b) Silicon and germanium detection efficiency as functions of energy.

shows all energy values for which the efficiency was measured. The accuracy of the efficiency curve is essential for spectrum correction. The silicon detector efficiency drops under 10\% above $30 \mathrm{keV}$, because of its small thickness and its low $Z$. It results in lower counting rates and a higher uncertainty in the efficiency calibration above $30 \mathrm{keV}$. Accounting for this, spectrum correction will be limited to energies below $40 \mathrm{keV}$. For germanium, measurements were made in air, and corrections for transmission lead to higher uncertainty on its efficiency fit in low energies [Fig. 5(b)].

Main phenomena that distort the emitted spectra below $50 \mathrm{keV}$ were characterized, but not all of them need to be corrected for. We intend to develop a mathematical model of the whole transfer function. However, in this first approach, we wanted to validate the correction method for efficiency loss and escape phenomenon by comparing two low-energy spectra obtained, respectively, with silicon and germanium detectors. 


\section{Spectra correction}

On the one hand, the main difficulty for spectra detected by the silicon diode is efficiency correction, which may result in biased high-energy counts. On the other hand, the germanium spectrum is biased in the low-energy range because of the escape peaks. In a continuous spectrum, as produced by medical $X$-ray tubes, a fraction of the counts are translated to lower energies. This can be clearly seen in Fig. 6 comparing two raw Mammograph spectra, especially on molybdenum $X_{K}$ rays (17.46 and $19.62 \mathrm{keV}$ ), and their associated escape peaks (for example 6.48 and $7.58 \mathrm{keV}$ for a PE peak at $17.46 \mathrm{keV}$ ).This X-ray tube has a molybdenum anode and molybdenum internal filtration of unknown thickness and is usually operated at $25 \mathrm{kV}$ and $100 \mathrm{~mA}$. Those spectra were measured at a 1-m distance from the beryllium window, with a tungsten collimator $(100-\mu \mathrm{m}$ diameter, $4 \mathrm{~mm}$ thick) located on top of the detector nose to prevent pile up (dead time $<0.5 \%$; counting rate $\sim 100 \mathrm{~s}^{-1}$ for both detectors). The electronic settings are described in Table 2. Because of the collimator size, alignment was critical and was therefore controlled by means of a motorized bench (rotation accuracy within $0.1^{\circ}$ ).

Efficiency was the only correction carried out on silicon spectrum, as shown in Fig. 7(a). Shaping times were low (3.2 and $0.2 \mu \mathrm{s}$ ) because our detector is only $500 \mu \mathrm{m}$ thick. Pile up was hardly occurring, and the counts increase in the high-energy range is due to our correction process: A few background counts remained above $25 \mathrm{keV}$ and were amplified ten times by the efficiency correction. However, counts in this area remain negligible compared with those below $25 \mathrm{keV}$ (log scale).

The germanium spectrum, in turn, was corrected first for escape phenomena and then for efficiency: The resulting spectra appear in Fig. 7(b).

Eventually, Fig. 8 presents the comparison of both corrected spectra. It is worth noticing that both spectra are similar in

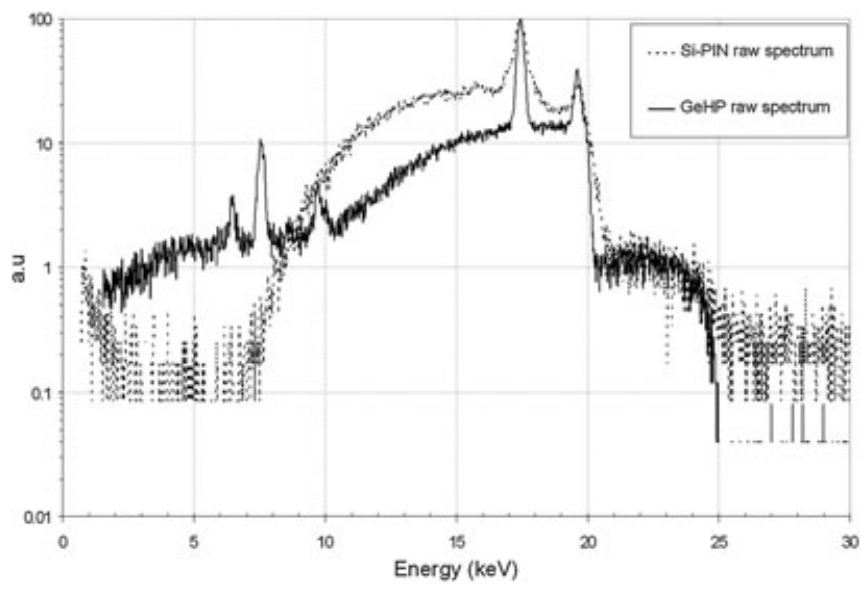

Figure 6. Detected spectra of mammography X-ray tube acquired with silicon and germanium detectors. general shape. For germanium, the mean energy value changes from $\bar{E}_{\text {non.corrected, } \mathrm{Ge}}=15.84 \mathrm{keV}$ to $\overline{\mathrm{E}}_{\text {corrected, } \mathrm{Ge}}=16.72 \mathrm{keV}$, even though the kilo-voltage peak remains constant: $\mathrm{kVp}$ Ge $=25.0$ $\mathrm{kV}$. For the silicon spectrum, the features measured are $\bar{E}_{n o n}$. corrected, $\mathrm{si}=15.85 \mathrm{keV}$ and $\overline{\mathrm{E}}_{\text {corrected, } \mathrm{si}}=17.19 \mathrm{keV}$ and $\mathrm{kVp} \mathrm{si}=$ $25.4 \mathrm{kV}$. Indeed, silicon efficiency drops for energies above $10 \mathrm{keV}$, explaining the change in the mean energy value. The $k V p$ value is less accurate, as expected, because of a worse resolution. Unfortunately, there is no reference spectrum to compare with our measured ones. Indeed, most spectrum simulation software does not calculate spectra for molybdenum anodes. Even though 'IPEM catalogue of diagnostic X-ray spectra' do calculate such spectra, it requires parameters such as anode angle and thickness of filtration that X-ray tube manufacturers do not supply. The same problem of geometry limits the accuracy of Monte Carlo simulations. The difference between corrected spectra is small and allows a validation of this escape correction method for X-ray beam study.

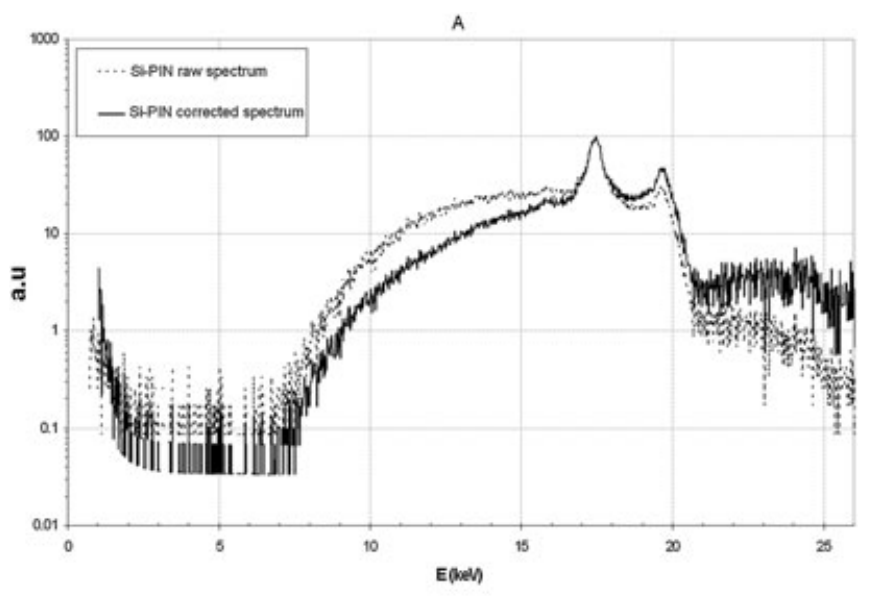

B

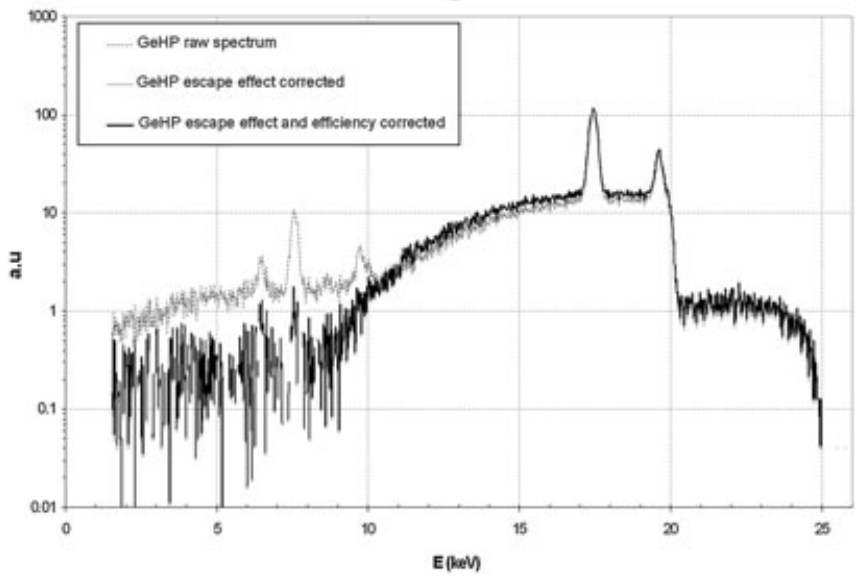

Figure 7. (a) and (b): Resp. silicon and germanium's Mammograph corrected spectra (dots for raw spectra and lines for corrected ones).

\begin{tabular}{|lcccccc|}
\hline \multicolumn{2}{|c|}{$\begin{array}{c}\text { Table 2. Collimator size and electronic settings of both detectors } \\
\text { Distance } \\
\text { source-detector }(\mathrm{m})\end{array}$} & $\begin{array}{c}\text { Tungsten collimator } \\
\text { diameter }(\mu \mathrm{m})\end{array}$ & $\begin{array}{c}\text { Collimator } \\
\text { thickness }(\mathrm{mm})\end{array}$ & $\begin{array}{c}\text { Channels } \\
\text { Live time }(\mathrm{s})\end{array}$ & $\begin{array}{c}\text { Rise time }(\mu \mathrm{s}) \\
\text { Flat } \\
\text { top }(\mu \mathrm{s})\end{array}$ \\
\hline Silicon & 1.00 & 100 & 4 & 4096 & 1115 & 3.2 \\
Germanium & 1.00 & 100 & 4 & 4096 & 2205 & 5.6 \\
\hline
\end{tabular}




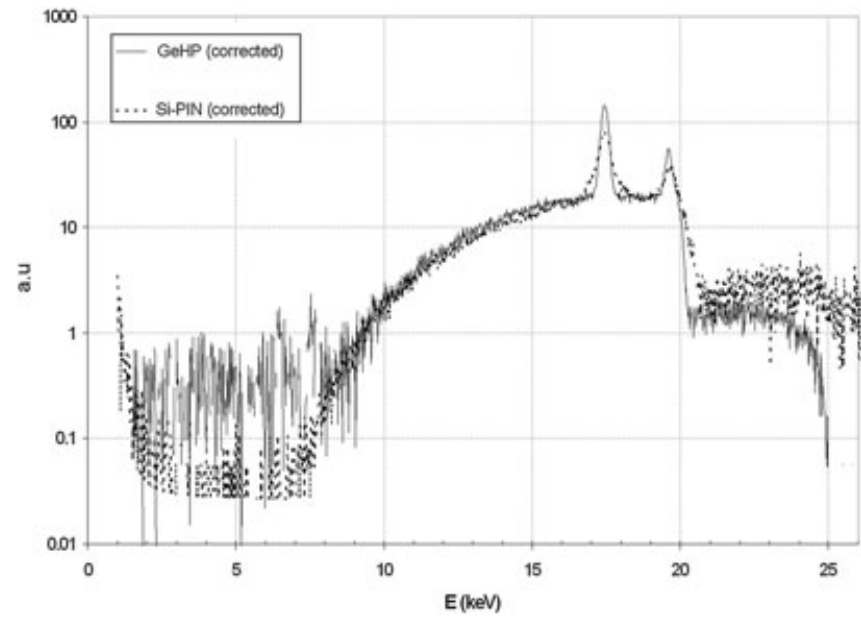

Figure 8. Comparison of corrected mammography X-ray tube spectra.

\section{Perspectives}

We are currently developing numerical models of our detectors, to run Monte Carlo simulations. This will assess the effect of physical phenomena on the spectrum, such as scattering in the collimator's head.

Subsequently, this work will be extended to energies above $50 \mathrm{keV}$ with a study of the Compton phenomenon. Then, a multi-parameter response function will be calculated from reference spectra, to correct measured medical X-ray tubes spectra for the detection system response function.

Moreover, another field of application of this set of detectors will be the study of sealed sources, as ${ }^{125}$ I seeds. Used in brachytherapy, those sources present a high activity (740 MBq) and many fluorescence peaks because of their complex design and multi- material composition.

As a final goal, the knowledge of the spectra will allow us to reduce dose delivery uncertainty and maybe to define a beam quality criterion to be compared with actual beam quality indices used in dosimetry of medium and low energy X-rays.

\section{Conclusions}

Calibration of a set of semiconductor detectors was conducted at LNHB, using a monochromatic X-ray source, SOLEX and calibrated radionuclides. This calibration allows us to correct low-energy spectra emitted by X-ray tubes for escape phenomenon and efficiency loss. We still have to take into account other interferences such as Gaussian widening and scattering in the collimator. However, this new approach combines the assets of germanium and silicon detectors, and comparison of both corrected spectra confirms the validity of germanium escape peaks correction.

Once the calibration finished, we aim at scanning various tubes and at measuring 2D beam profiles. High flux and tiny collimators require a precise alignment between the tube's focal and the collimated detector head; therefore, a dedicated four-axis motorized bench is being set up at LNHB. Another set of two motorized translation stages allows the scanning of the detector crystal by moving the collimator in front of the detector's window, to optimize the centring of the beam on the crystal. A dedicated piece of software will run the acquisition, calculate corrected spectra and give back the tube's characteristics $(\bar{E}, k V p)$, to compare them with other beam indicators such as the half value layer.

\section{Acknowledgements}

CEA-LNHB-LMA is gratefully acknowledged for providing calibration sources and access to the SOLEX facility.

\section{References}

[1] C. Bonnelle et al. Nucl. Instrum. Methods Phys. Res. A 2004, 516, 594.

[2] J. Plagnard, C. Bobin, M. C. Lépy. X-Ray Spectrom. 2007, 36, 191.

[3] Y. Menesguen, M. C. Lépy. Nucl. Instrum. Methods Phys. Res. A 2012, 695, 193.

[4] H. Ruellan, M. C. Lépy. Nucl. Instrum. Methods Phys. Res. A 1996, 369, 651.

[5] G. W. Phillips, K. M. Marlow. Nucl. Instrum. Methods Phys. Res. 1976, 137, 525.

[6] J. Lacer, E. Haller, R. C. Cordi. IEEE Trans. Nucl. Sci. 1977, 24, 53.

[7] M. P. Fioratti, S. R. Piermattei. Nucl. Instrum. Methods Phys. Res. 1971, 96, 605 .

[8] S. J. B. Reed, R. D. Ware. J. Phys. E: Sci. Instrum. 1976, 9, 1023.

[9] L. H. Christensen. X-Ray Spectrom. 1979, 8, 146. 